$\overline{\text { Original }}$

\title{
Microbial Contamination in Home Laundry Operations in Japan
}

\author{
ATSUSHI TABATA, DAXIN ZHANG, TAKUYA MAEDA, \\ HIDEAKI NAGAMUNE, AND HIROKI KOURAI* \\ Department of Biological Science and Technology, Faculty of Engineering, The \\ University of Tokushima, Tokushima 770-8506, Japan \\ Received 23 March 2002/Accepted 27 May 2002
}

\begin{abstract}
The hygiene levels in home laundry operations in Japan have been changing with the changes in lifestyle. Microbial transmission through home laundry has been reported, but mainly in North American and European countries. The home laundering situation in Japan is unique among many countries, and the most characteristic point is that Japanese use the leftover bath water for laundering the next day. However, such a unique custom may cause household microbial transmission. In order to clarify the link between the microbial transmission and the home laundering operation in Japan, a bacteriological investigation of various articles was done. Children's underpants, bath towels, kitchen rags, the washing machine itself and the leftover bath water were investigated. Although variations in bacterial strains were seen, many Staphylococcus spp. were isolated from every sample. Although pathogenic strains were not detected, some opportunistic pathogens were detected. Furthermore, since many bacteria were isolated from bath water, home laundering operations with the used bath water may cause microbial contamination of the laundry.
\end{abstract}

Key words : Home laundry/Japan/Microbial contamination/Microbial transmission/Bath water.

\section{INTRODUCTION}

In 1998 the World Health Organization (WHO) declared that infectious diseases, which include AIDS and tuberculosis, are the most common cause of morbidity worldwide (World Hearth Organization, 1998), and this situation is greatly influenced by the change in social conditions (Sattar et al., 1999). Even when various antibiotics are used now, microbial infection is the most common cause of morbidity. It was indicated that approximately $50 \%$ of so-called hospital infections were actually acquired in the community, prior to hospitalization (Meer et al., 1981), and when minor infections that do not result in medical consultations are also taken into consideration, the risk of microbial infection in our living environment is high. In such a situation, increasing attention has been paid to home

*Corresponding author. Tel: +81-88-656-7408, Fax : +8188-656-9148. hygiene in recent years, and many studies have been performed about the relation between home hygiene and microbial transmission (Davis et al., 1968; Enriquez et al., 1997; Finch et al., 1978; Larson, 1999; Larson and Duarte, 2001; Scott, 1996, 1999; Scott et al., 1982; Terpstra, 1998; Zhao et al., 1998).

Within the home environment, there are many opportunities for microbial exposure and transmission. Some studies have also described the link between microbial transmission and laundering operations (Barrie et al., 1994; Belkin, 2001; Blaser, 1984; Buford et al., 1977; Datta and Pridie, 1960; Larson and Duarte, 2001; Oliphant et al., 1949; Sidwell et al., 1971; Standaert et al., 1994; Walter and Schillinger, 1975; Wiksell et al., 1973). However, these studies have mainly been done in North American or European countries, and there is little information on laundering operations in Japan.

There are various points regarding the home laundering operation in Japan that deserve further 
attention. For example, shorter laundering times, lower washing temperatures, little water usage and lower detergent concentrations may all reduce the hygienic benefits of laundering. These laundering conditions in Japan are greatly related to the change in the social situation. In the first place, it is thought that the above-mentioned laundry conditions reflect the desire to economize. As the most striking example of economizing, it is common for many families to use the leftover bath water to do the next day's laundry for the purpose of saving water. The use of the leftover bath water to do the laundry has declined from the past, but about $30 \%$ or less of families still use it. However it is thought that many microbes will exist in the leftover bath water and laundering with it may cause microbial transmission. Second, the laundering frequency has decreased because the number of nuclear families or solitary dwellers has increased. Third, since the women have been spending more time at work, they have been unable to spend sufficient time for laundering operations. The practice of drying the clothes inside the homes has also become more widespread for the same reason. In addition to the change in these situations, the laundry does not become dry enough because the climate of Japan is so humid. There is a high possibility that microbes not removed by laundering operations may flourish. These laundering situations may cause not only inadequate cleaning but also the opportunity for clean clothes or the washing machine to become cross-contaminated.

The present study was performed to clarify the link between the risk of household microbial transmission and home laundering operations in Japan by investigating the microbial contamination of various articles (children's underpants, used bath towels and kitchen rags), the washing machine and bath water.

\section{MATERIALS AND METHODS}

\section{Selection agar}

Standard method agar (Nissui Pharmaceutical, Tokyo) was used to count the viable cell number. In order to isolate the bacteria from the samples, eight kinds of selection agars were used. Coliform bacteria were assayed on Desoxycholate agar (Nissui) and Escherichia coli on Lauryl sulfate MUG broth (Nissui) containing 1.5\% agar. Salmonella spp., Shigella spp., Serratia spp. and Pseudomonas spp. were assayed on Brilliant green agar (Oxoid, Basingstoke, Hampshire, England), SS agar (Nissui), DNA agar (Nissui) and NAC agar (Nissui) respectively. Staphylococcus spp. was assayed on Staphylococcus medium agar (Nissui) and Bacillus spp. (mainly for Bacillus cereus) on NGKG agar base
(Nissui) containing $1.8 \%$ egg yolk emulsion (OXOID).

\section{Detection and quantitation of bacteria on se- lected garments}

Nine pairs of underpants worn by children (aged 45 years) were provided by nine volunteer families. The samples were collected in a nylon bag and transported to our laboratory without being cooled. These samples were washed by being rubbed for $3 \mathrm{~min}$ in stomacher polyethylene bag containing $500 \mathrm{ml}$ of icecooled sterile physiological saline $(0.85 \% \mathrm{NaCl})$ with $0.2 \%$ Tween 80 . Nine bath towels were also provided by nine volunteer families. The samples were collected in a nylon bag and transported to our laboratory without being cooled or dried. $100 \mathrm{~cm}^{2}(10 \times 10$ $\mathrm{cm}$ ) pieces from the center region of each bath towel were cut out with sterile scissors. Cut samples were washed by rubbing for $3 \mathrm{~min}$ in a stomacher polyethylene bag containing $500 \mathrm{ml}$ of ice-cooled sterile physiological saline $(0.85 \% \mathrm{NaCl})$ with $0.2 \%$ Tween 80 . Ten used kitchen rags were provided by ten volunteer families. The samples were collected in a polyethylene bag and transported to our laboratory without being cooled or dried. $100 \mathrm{~cm}^{2}(10 \times 10 \mathrm{~cm})$ pieces from the center region of each kitchen rag were cut out with sterile scissors. Cut samples were washed by rubbing for $3 \mathrm{~min}$ in a stomacher polyethylene bag containing $500 \mathrm{ml}$ of ice-cooled sterile physiological saline $(0.85 \% \mathrm{NaCl})$ with $0.2 \%$ Tween $80.0 .5 \mathrm{ml}$ of each sample solution was added to the $4.5 \mathrm{ml}$ of icecooled sterile $0.3 \mathrm{mM}$ phosphate buffer and the solution was diluted in steps.

$0.1 \mathrm{ml}$ of these dilutions was spread on Standard method agar. Another $0.1 \mathrm{ml}$ was also spread on various selection agars to count the selective cell number. In order to identify the Bacillus spp., the diluted sample solution was heated $\left(80^{\circ} \mathrm{C}, 10 \mathrm{~min}\right)$ and $0.1 \mathrm{ml}$ was spread on Standard method agar after being cooled at room temperature. These spread agar plates were incubated for $24 \mathrm{~h}$ (or $48 \mathrm{~h}$ ) at $37^{\circ} \mathrm{C}$ and colonies were counted.

\section{Detection and quantitation of bacteria from the washing machine}

Five swabs of the drum and five swabs of the drain ditch from the washing machine were provided by five volunteer families. Sterile nil-bleached gauze $(5 \times 5$ $\mathrm{cm}, 4$ piles) was pre-wetted with $10 \mathrm{ml}$ of sterile $0.3 \mathrm{mM}$ phosphate buffer, and the areas on the middle region of drum $\left(100 \mathrm{~cm}^{2}\right)$ and drain ditch $\left(100 \mathrm{~cm}^{2}\right)$ were swabbed for $1 \mathrm{~min}$ by hands covered with sterile polyethylene gloves. The samples were collected in a polyethylene bag and transported to our laboratory 
without being cooled or dried. These samples were washed by rubbing for $3 \mathrm{~min}$ in a stomacher polyethylene bag containing $500 \mathrm{ml}$ of ice-cooled sterile physiological saline $(0.85 \% \mathrm{NaCl})$ with $0.2 \%$ Tween 80 . These sample solutions were diluted and spread as mentioned above.

\section{Detection and quantitation of bacteria in bath wa- ter}

Ten bath water samples were provided by ten volunteer families. Used bath water was collected in sterilized bottles after the water had been left standing for $12 \mathrm{~h}$. The samples were transported to our laboratory without being cooled. These sample solutions were diluted and spread as mentioned above.

\section{Identification of isolated bacteria by API system}

Colonies grown on a various selection agars were discriminated visually and further underwent the oxydase test, Gram-stain and microscopic observation. Isolated bacteria were identified by API system (bioMerieux Industry, Tokyo). API 20E was used for identification of coliform bacteria, Escherichia coli, Salmonella spp., Shigella spp., Serratia spp. and Pseudomonas spp. and so on. API STAPH was used for Staphylococcus spp. and API $50 \mathrm{CH}$ for Bacillus spp.. The method followed the API manual and results with over $60 \%$ accuracy were adopted.

\section{RESULTS}

\section{Occurrence of bacteria on children's underpants}

Bacteria isolated from children's underpants are listed in Table 1. Many bacteria were isolated although no samples had any visible fecal contamination. The most frequently isolated species was Staphylococcus spp. $\left(5.7 \times 10^{2}-5.0 \times 10^{4} \mathrm{CFU} / \mathrm{cm}^{2}\right)$. The next was Bacillus spp. $\left(1.7 \times 10^{1}-3.3 \times 10^{4} \mathrm{CFU}\right.$ $\left(\mathrm{cm}^{2}\right)$, and it was isolated from 7 of the 9 samples. Coliform bacteria and Pseudomonas spp. were also isolated from 6 of the 9 samples and 5 of the 9 samples, respectively. E. coli was isolated in only 2 of the 9 samples, and the number was small at $1.3 \times 10^{1}-$ $1.7 \times 10^{1} \mathrm{CFU} / \mathrm{cm}^{2}$. The enteric pathogens, Salmonella spp. and Shigella spp., were not detected.

\section{Occurrence of bacteria on bath towels}

Bacteria isolated from bath towels are listed in Table 2. The number of isolated bacteria differed largely in each sample. Many bacteria were isolated from samples that emitted a nasty smell or were soiled heavily. Staphylococcus spp. was isolated from all samples $\left(4.5 \times 10^{1}-5.6 \times 10^{4} \mathrm{CFU} / \mathrm{cm}^{2}\right)$ as it was from the children's underpants, and it was the predominant strain that was isolated from all bath towels. The next frequently isolated species was coliform bacteria $\left(2.8 \times 10^{2}-2.0 \times 10^{4} \mathrm{CFU} / \mathrm{cm}^{2}\right)$, and there was comparatively little Bacillus spp. (2 of the 9 samples) isolated. Micrococcus spp., which was not isolated from children's underpants, and Serratia spp.,

TABLE 1. Bacteria isolated from children's underpants.

\begin{tabular}{ccccccc}
\hline & $\begin{array}{c}\text { Total } \\
\text { Seterotrophic } \\
\text { slate count } \\
\left(\text { CFU } / \mathrm{cm}^{2}\right)\end{array}$ & $\begin{array}{c}\text { Bacterial count } \\
\left(\text { CFU } / \mathrm{cm}^{2}\right)\end{array}$ \\
\cline { 3 - 7 } & $\begin{array}{c}\text { Coliform } \\
\text { bacteria }^{b}\end{array}$ & $\begin{array}{c}\text { Escherichia } \\
\text { coli }\end{array}$ & $\begin{array}{c}\text { Pseudomonas } \\
\text { spp. }\end{array}$ & $\begin{array}{c}\text { Staphylococcus } \\
\text { spp. }\end{array}$ & $\begin{array}{c}\text { Bacillus } \\
\text { spp. }\end{array}$ \\
\hline $\mathrm{A}$ & $8.3 \times 10^{2}$ & $1.7 \times 10^{1}$ & $1.3 \times 10^{1}$ & $\mathrm{ND}^{a}$ & $8.0 \times 10^{2}$ & $1.7 \times 10^{1}$ \\
$\mathrm{~B}$ & $6.7 \times 10^{3}$ & $1.7 \times 10^{1}$ & $\mathrm{ND}$ & $1.7 \times 10^{1}$ & $2.8 \times 10^{3}$ & $\mathrm{ND}$ \\
$\mathrm{C}$ & $1.9 \times 10^{3}$ & $3.3 \times 10^{2}$ & $\mathrm{ND}$ & $\mathrm{ND}$ & $5.7 \times 10^{2}$ & $\mathrm{ND}$ \\
$\mathrm{D}$ & $7.3 \times 10^{2}$ & $\mathrm{ND}$ & $1.7 \times 10^{1}$ & $2.1 \times 10^{2}$ & $1.5 \times 10^{3}$ & $5.3 \times 10^{2}$ \\
$\mathrm{E}$ & $5.7 \times 10^{3}$ & $\mathrm{ND}$ & $\mathrm{ND}$ & $3.3 \times 10^{1}$ & $3.3 \times 10^{3}$ & $3.3 \times 10^{1}$ \\
$\mathrm{~F}$ & $9.0 \times 10^{3}$ & $1.7 \times 10^{1}$ & $\mathrm{ND}$ & $9.3 \times 10^{1}$ & $1.6 \times 10^{3}$ & $1.3 \times 10^{2}$ \\
$\mathrm{G}$ & $3.3 \times 10^{3}$ & $\mathrm{ND}$ & $\mathrm{ND}$ & $\mathrm{ND}$ & $3.0 \times 10^{3}$ & $1.7 \times 10^{2}$ \\
$\mathrm{H}$ & $5.7 \times 10^{5}$ & $1.7 \times 10^{1}$ & $\mathrm{ND}$ & $\mathrm{ND}$ & $5.0 \times 10^{4}$ & $3.3 \times 10^{4}$ \\
I & $4.7 \times 10^{3}$ & $1.4 \times 10^{3}$ & $\mathrm{ND}$ & $3.7 \times 10^{2}$ & $7.7 \times 10^{2}$ & $2.0 \times 10^{2}$ \\
\hline Arithmetic & $6.7 \times 10^{4}$ & $3.0 \times 10^{2}$ & $1.5 \times 10^{1}$ & $1.4 \times 10^{2}$ & $7.1 \times 10^{3}$ & $4.9 \times 10^{3}$ \\
ave. & & & & & & \\
Geometric & $5.3 \times 10^{3}$ & $5.8 \times 10^{1}$ & $1.5 \times 10^{1}$ & $8.3 \times 10^{1}$ & $2.2 \times 10^{3}$ & $2.4 \times 10^{2}$ \\
ave. & & & & & & \\
\hline
\end{tabular}

${ }^{a}$ Not detected.

${ }^{b}$ Coliform bacteria consisted of Pantoea spp.

The following were not isolated from the underpants: Salmonella spp., Shigella spp., Serratia spp., Yersinia spp., Aeromonas spp., Acinetobacter spp., Chryseobacterium spp., Alcaligenes spp. and Micrococcus spp. 
TABLE 2. Bacteria isolated from bath towels.

\begin{tabular}{|c|c|c|c|c|c|c|c|}
\hline \multirow{2}{*}{ Sample } & \multirow{2}{*}{$\begin{array}{c}\text { Total } \\
\text { heterotrophic } \\
\text { plate count } \\
\left(\mathrm{CFU} / \mathrm{cm}^{2}\right)\end{array}$} & \multicolumn{6}{|c|}{$\begin{array}{l}\text { Bacterial count } \\
\left(\mathrm{CFU} / \mathrm{cm}^{2}\right)\end{array}$} \\
\hline & & $\begin{array}{l}\text { Coliform } \\
\text { bacteria }^{b}\end{array}$ & $\begin{array}{l}\text { Serratia } \\
\text { spp. }\end{array}$ & $\begin{array}{c}\text { Pseudomonas } \\
\text { spp. }\end{array}$ & $\begin{array}{l}\text { Micrococcus } \\
\text { spp. }\end{array}$ & $\begin{array}{c}\text { Staphylococcus } \\
\text { spp. }\end{array}$ & $\begin{array}{c}\text { Bacillus } \\
\text { spp. }\end{array}$ \\
\hline A & $1.1 \times 10^{4}$ & $N D^{a}$ & ND & ND & ND & $1.2 \times 10^{3}$ & ND \\
\hline B & $1.0 \times 10^{5}$ & $2.0 \times 10^{4}$ & ND & $1.1 \times 10^{4}$ & ND & $5.6 \times 10^{4}$ & ND \\
\hline C & $7.5 \times 10^{1}$ & ND & ND & $2.0 \times 10^{1}$ & ND & $4.5 \times 10^{1}$ & ND \\
\hline D & $2.3 \times 10^{5}$ & $6.0 \times 10^{2}$ & ND & ND & ND & $2.4 \times 10^{4}$ & ND \\
\hline$E$ & $5.9 \times 10^{2}$ & ND & ND & ND & ND & $5.6 \times 10^{3}$ & ND \\
\hline $\mathrm{F}$ & $5.8 \times 10^{6}$ & $1.5 \times 10^{4}$ & $5.0 \times 10^{2}$ & $1.6 \times 10^{3}$ & $1.3 \times 10^{4}$ & $2.9 \times 10^{4}$ & ND \\
\hline G & $1.2 \times 10^{3}$ & $1.5 \times 10^{2}$ & ND & $5.0 \times 10^{1}$ & ND & $2.8 \times 10^{3}$ & $3.0 \times 10^{2}$ \\
\hline $\mathrm{H}$ & $1.2 \times 10^{5}$ & $6.5 \times 10^{3}$ & ND & $2.8 \times 10^{2}$ & ND & $4.9 \times 10^{3}$ & $1.0 \times 10^{3}$ \\
\hline 1 & $1.3 \times 10^{3}$ & $2.8 \times 10^{2}$ & ND & ND & ND & $1.1 \times 10^{3}$ & ND \\
\hline $\begin{array}{c}\text { Arithmetic } \\
\text { ave. }\end{array}$ & $7.0 \times 10^{5}$ & $7.1 \times 10^{3}$ & $5.0 \times 10^{2}$ & $2.6 \times 10^{3}$ & $1.3 \times 10^{4}$ & $1.4 \times 10^{4}$ & $6.5 \times 10^{2}$ \\
\hline $\begin{array}{c}\text { Geometric } \\
\text { ave. }\end{array}$ & $1.3 \times 10^{5}$ & $1.9 \times 10^{3}$ & $5.0 \times 10^{2}$ & $3.5 \times 10^{2}$ & $1.3 \times 10^{4}$ & $3.8 \times 10^{3}$ & $5.5 \times 10^{2}$ \\
\hline
\end{tabular}

${ }^{a}$ Not detected.

${ }^{\circ}$ Coliform bacteria consisted of Klebsiella spp., Enterobacter spp. and Pantoea spp.

The following were not isolated from bath towels: Salmonella spp., Shigella spp., Escherichia coli, Yersinia spp., Aeromonas spp., Acinetobacter spp., Chryseobacterium spp. and Alcaligenes spp.

TABLE 3. Bacteria isolated from kitchen rags.

\begin{tabular}{|c|c|c|c|c|c|c|c|c|c|}
\hline \multirow[b]{2}{*}{ Sample } & \multirow{2}{*}{$\begin{array}{c}\text { Total } \\
\text { heterotrophic- } \\
\text { plate count } \\
\left(\mathrm{CFU} / \mathrm{cm}^{2}\right)\end{array}$} & \multicolumn{8}{|c|}{$\begin{array}{l}\text { Bacterial count } \\
\left(\mathrm{CFU} / \mathrm{cm}^{2}\right)\end{array}$} \\
\hline & & $\begin{array}{l}\text { Coliform } \\
\text { bacteria }^{b}\end{array}$ & $\begin{array}{c}\text { Escheri- } \\
\text { chia } \\
\text { coli }\end{array}$ & $\begin{array}{l}\text { Serratia } \\
\text { spp. }\end{array}$ & $\begin{array}{c}\text { Aero- } \\
\text { monas } \\
\text { spp. }\end{array}$ & $\begin{array}{c}\text { Pseudo- } \\
\text { monas } \\
\text { spp. }\end{array}$ & $\begin{array}{c}\text { Alcali- } \\
\text { genes } \\
\text { spp. }\end{array}$ & $\begin{array}{l}\text { Staphylo- } \\
\text { coccus } \\
\text { spp. }\end{array}$ & $\begin{array}{l}\text { Bacillus } \\
\text { spp. }\end{array}$ \\
\hline A & $1.7 \times 10^{6}$ & $2.2 \times 10^{5}$ & $\mathrm{ND}^{a}$ & $4.2 \times 10^{4}$ & $1.4 \times 10^{5}$ & $8.8 \times 10^{3}$ & ND & $9.2 \times 10^{5}$ & $4.0 \times 10^{3}$ \\
\hline B & $8.2 \times 10^{6}$ & $2.3 \times 10^{5}$ & $1.5 \times 10^{5}$ & $1.3 \times 10^{4}$ & $1.1 \times 10^{5}$ & $1.1 \times 10^{4}$ & $2.0 \times 10^{4}$ & $4.2 \times 10^{5}$ & $1.3 \times 10^{4}$ \\
\hline C & $1.4 \times 10^{5}$ & $1.3 \times 10^{4}$ & ND & $3.1 \times 10^{4}$ & $6.5 \times 10^{4}$ & $1.5 \times 10^{4}$ & ND & $3.2 \times 10^{3}$ & $5.0 \times 10^{1}$ \\
\hline D & $9.4 \times 10^{4}$ & $4.5 \times 10^{2}$ & ND & ND & ND & ND & $5.8 \times 10^{2}$ & $1.8 \times 10^{4}$ & ND \\
\hline $\mathrm{E}$ & $2.7 \times 10^{3}$ & $3.0 \times 10^{2}$ & ND & ND & $5.0 \times 10^{1}$ & ND & $1.5 \times 10^{2}$ & $3.0 \times 10^{2}$ & $5.0 \times 10^{1}$ \\
\hline $\mathrm{F}$ & $1.1 \times 10^{4}$ & $3.5 \times 10^{2}$ & ND & $3.0 \times 10^{2}$ & $1.0 \times 10^{3}$ & ND & $1.0 \times 10^{3}$ & $7.5 \times 10^{3}$ & ND \\
\hline G & $1.0 \times 10^{5}$ & $9.6 \times 10^{3}$ & ND & $1.1 \times 10^{3}$ & $2.0 \times 10^{3}$ & $6.0 \times 10^{2}$ & $5.0 \times 10^{2}$ & $2.8 \times 10^{4}$ & $3.8 \times 10^{2}$ \\
\hline $\mathrm{H}$ & $2.8 \times 10^{6}$ & $1.4 \times 10^{3}$ & ND & ND & ND & ND & $2.0 \times 10^{3}$ & $2.5 \times 10^{4}$ & $1.5 \times 10^{2}$ \\
\hline 1 & $4.4 \times 10^{6}$ & $1.6 \times 10^{5}$ & ND & $4.2 \times 10^{4}$ & $2.0 \times 10^{5}$ & $1.2 \times 10^{4}$ & $8.8 \times 10^{3}$ & $3.6 \times 10^{6}$ & $1.4 \times 10^{3}$ \\
\hline $\mathrm{J}$ & $1.4 \times 10^{7}$ & $3.4 \times 10^{4}$ & ND & $5.0 \times 10^{2}$ & $1.9 \times 10^{4}$ & $4.0 \times 10^{2}$ & $4.5 \times 10^{3}$ & $1.1 \times 10^{6}$ & $3.9 \times 10^{4}$ \\
\hline $\begin{array}{c}\text { Arithmetic } \\
\text { ave. }\end{array}$ & $3.1 \times 10^{6}$ & $6.7 \times 10^{4}$ & $1.5 \times 10^{5}$ & $1.9 \times 10^{4}$ & $6.7 \times 10^{4}$ & $8.0 \times 10^{3}$ & $4.7 \times 10^{3}$ & $6.1 \times 10^{5}$ & $7.3 \times 10^{3}$ \\
\hline $\begin{array}{c}\text { Geometric } \\
\text { ave. }\end{array}$ & $4.0 \times 10^{5}$ & $6.8 \times 10^{3}$ & $1.5 \times 10^{5}$ & $5.3 \times 10^{3}$ & $1.2 \times 10^{4}$ & $4.0 \times 10^{3}$ & $1.7 \times 10^{3}$ & $5.2 \times 10^{4}$ & $8.9 \times 10^{2}$ \\
\hline
\end{tabular}

${ }^{a}$ Not detected.

${ }^{b}$ Coliform bacteria consisted of Klebsiella spp., Enterobacter spp. and Pantoea spp.

The following were not isolated from the kitchen rags: Salmonella spp., Shigella spp., Yersinia spp., Acinetobacter spp., Chryseobacterium spp. and Micrococcus spp.

which is an opportunistic pathogen, were isolated from the bath towel harboring the highest amount of recoverable bacteria. Pseudomonas spp. was isolated from more than half of the samples ( 5 of the 9 samples). E. coli, Salmonella spp., and Shigella spp. were not detected.

\section{Occurrence of bacteria on kitchen rags}

Bacteria isolated from kitchen rags are listed in Table 3. The number of isolated bacteria differed largely from sample to sample. Staphylococcus spp. was isolated from all samples $\left(3.0 \times 10^{2}-3.6 \times 10^{6}\right.$ $\mathrm{CFU} / \mathrm{cm}^{2}$ ) like they were from children's underpants and bath towels, and coliform bacteria was also 
isolated from all samples $\left(3.0 \times 10^{2}-2.3 \times 10^{5} \mathrm{CFU} /\right.$ $\left.\mathrm{cm}^{2}\right)$. In addition, Aeromonas spp. $\left(5.0 \times 10^{1}-2.0 \times\right.$ $\left.10^{5} \mathrm{CFU} / \mathrm{cm}^{2}\right)$, Bacillus spp. $\left(5.0 \times 10^{1}-3.9 \times 10^{4} \mathrm{CFU}\right.$ $\left./ \mathrm{cm}^{2}\right)$ and Serratia spp. $\left(3.0 \times 10^{2}-4.2 \times 10^{4} \mathrm{CFU} /\right.$ $\mathrm{cm}^{2}$ ) were detected. Pseudomonas spp. was isolated from half of the samples (5 of the 10 samples), just as it had been from children's underpants and bath towels. Salmonella spp. and Shigella spp. were not detected.

The conditions for using and washing the kitchen rags are shown in Table 4 . The reason for this investigation is that these conditions may influence the number of isolated bacteria. It was revealed that detergent was used to wash the kitchen rags in almost all homes, but sterilization by boiling was rarely done. Clear correlation was not seen between the number of isolated bacteria and the period and frequency of use or the washing method used.

\section{Occurrence of bacteria in washing machines}

Bacteria isolated from the drums and drain ditches of five washing machines are listed in Table 5 and 6 , respectively. Some bacteria were isolated from all drain ditch samples, whereas some of the drum samples did not have any detectable bacteria. It is obvious that the distribution of bacteria changes with the part of the washing machine that is tested even if samples are from the same machine. As a whole, Gram-positive bacteria were predominantly isolated, the most frequently isolated species was Staphylococcus spp. $\left(5.0 \times 10^{1}-1.9 \times 10^{4} \mathrm{CFU} / \mathrm{cm}^{2}\right)$ and the next was Micrococcus spp. $\left(5.0 \times 10^{1}-1.8\right.$ $\left.\times 10^{5} \mathrm{CFU} / \mathrm{cm}^{2}\right)$. Many coliform bacteria and Staphylococcus spp. were isolated from the drain ditch samples, and Aeromonas spp. and Yersinia spp. were isolated only from drain ditch samples. E. coli, Salmonella spp., Shigella spp., Serratia spp. and Bacillus spp. were not detected.
Information about how long the machine had been used, the type of machine it was and the washing conditions are shown in Table 7. The reason for this investigation is that the condition of the washing machine and washing conditions may influence the number of bacteria which are isolated. Although significant correlation was not seen between the numbers of isolated bacteria and the period of use, if the attention is paid to the type of machine, it was suggested that there were fewer bacteria in the automatic washing machine than in the manual washing machine. It was obvious that the number of isolated bacteria increased as the operation time was longer.

\section{Occurrence of bacteria in bath water}

Bacteria isolated from bath water are listed in Table 8. The number of isolated bacteria differed largely in each sample, and no bacteria were detected from 2 samples contrary to our expectation. It was a unique result that Alcaligenes spp. was the predominantly isolated species from bath water $\left(1.2 \times 10^{1}-1.1 \times\right.$ $\left.10^{6} \mathrm{CFU} / \mathrm{ml}\right)$. Many coliform bacteria $(<10-1.5 \times$ $\left.10^{3} \mathrm{CFU} / \mathrm{ml}\right)$ and Staphylococcus spp. $\left(1.0 \times 10^{1}-3.4\right.$ $\times 10^{4} \mathrm{CFU} / \mathrm{ml}$ ) were also isolated. Chryseobacterium spp. and Yersinia spp, which may show pathogenicity, were isolated from 5 of the 10 samples and 3 of the 10 samples, respectively. The incidence of $E$. coli isolation was considerably lower than we had expected (2 of the 10 samples). Salmonella spp., Shigella spp. and Serratia spp. were not detected.

Information about the composition of the volunteer families and the use of bathing additives is shown in Table 9. The reason for this investigation is that the composition of the family will influence the number of isolated bacteria. In addition to that, since some bathing additives may act as a disinfectant, the cases in which bathing additives were added or not were also investigated. These results showed good correlation

TABLE 4. Conditions related to using and washing the kitchen rags.

\begin{tabular}{ccccccc}
\hline \multirow{2}{*}{ Sample } & \multicolumn{2}{c}{ Conditions of use } & \multicolumn{3}{c}{ Washing conditions $^{a}$} \\
\cline { 2 - 6 } & Period of use & $\begin{array}{c}\text { Frequency of use } \\
\text { per day (times) }\end{array}$ & Water only & Detergent wash & Boiling \\
\hline A & 1 wk. & 4 & - & + & - \\
B & (No information) & 2 & - & + & - \\
C & 1 wk. & 3 & - & + & - \\
D & (No information) & 3 & - & + & - \\
E & 6 mo. & 6 & - & + & - \\
F & 1 mo. & 4 & - & - & - \\
G & 2 mo. & 15 & - & + & $(+)$ \\
H & 1 mo. & 8 & - & + & - \\
I & 2 d. & 7 & - & - & - \\
J & 1 mo. & 3 & - & & \\
\hline
\end{tabular}

${ }^{{ }^{-}}-$, Not performed; + , practiced; (+), sometimes practiced. 
TABLE 5. Bacteria isolated from the drum of the washing machine.

\begin{tabular}{|c|c|c|c|c|c|c|c|}
\hline \multirow{2}{*}{ Sample } & \multirow{2}{*}{$\begin{array}{c}\text { Total } \\
\text { heterotrophic } \\
\text { plate count } \\
\left(\mathrm{CFU} / \mathrm{cm}^{2}\right)\end{array}$} & \multicolumn{6}{|c|}{$\begin{array}{l}\text { Bacterial count } \\
\left(\mathrm{CFU} / \mathrm{cm}^{2}\right)\end{array}$} \\
\hline & & $\begin{array}{l}\text { Coliform } \\
\text { bacteria }^{b}\end{array}$ & $\begin{array}{l}\text { Yersinia } \\
\text { spp. }\end{array}$ & $\begin{array}{l}\text { Pseudomonas } \\
\text { spp. }\end{array}$ & $\begin{array}{l}\text { Alcaligenes } \\
\text { spp. }\end{array}$ & $\begin{array}{l}\text { Micrococcus } \\
\text { spp. }\end{array}$ & $\begin{array}{c}\text { Staphylococcus } \\
\text { spp. }\end{array}$ \\
\hline$A$ & $2.8 \times 10^{4}$ & $1.9 \times 10^{3}$ & $N^{a}$ & ND & ND & $5.0 \times 10^{1}$ & $7.0 \times 10^{3}$ \\
\hline B & $3.0 \times 10^{4}$ & ND & ND & ND & ND & $1.9 \times 10^{4}$ & ND \\
\hline C & ND & ND & ND & ND & ND & ND & ND \\
\hline D & $2.8 \times 10^{3}$ & ND & ND & $5.0 \times 10^{1}$ & ND & $7.0 \times 10^{2}$ & $5.5 \times 10^{2}$ \\
\hline $\mathrm{E}$ & ND & ND & ND & ND & ND & ND & ND \\
\hline $\begin{array}{c}\text { Arithmetic } \\
\text { ave. }\end{array}$ & $2.0 \times 10^{4}$ & $1.9 \times 10^{3}$ & - & $5.0 \times 10^{1}$ & - & $6.6 \times 10^{3}$ & $3.8 \times 10^{3}$ \\
\hline $\begin{array}{c}\text { Geometric } \\
\text { ave. }\end{array}$ & $1.3 \times 10^{4}$ & $1.9 \times 10^{3}$ & - & $5.0 \times 10^{1}$ & - & $8.7 \times 10^{2}$ & $2.0 \times 10^{3}$ \\
\hline
\end{tabular}

${ }^{a}$ Not detected.

${ }^{b}$ Coliform bacteria consisted of Pantoea spp.

The following were not isolated from the washing machine drum by swabbing: Salmonella spp., Shigella spp., Escherichia coli, Serratia spp., Aeromonas spp., Acinetobacter spp., Chryseobacterium spp. and Bacillus spp.

TABLE 6. Bacteria isolated from the drain ditch of the washing machine.

\begin{tabular}{|c|c|c|c|c|c|c|c|}
\hline \multirow{2}{*}{ Sample } & \multirow{2}{*}{$\begin{array}{c}\text { Total } \\
\text { heterotrophic } \\
\text { plate count } \\
\left(\mathrm{CFU} / \mathrm{cm}^{2}\right)\end{array}$} & \multicolumn{6}{|c|}{$\begin{array}{l}\text { Bacterial count } \\
\left(\mathrm{CFU} / \mathrm{cm}^{2}\right)\end{array}$} \\
\hline & & $\begin{array}{l}\text { Coliform } \\
\text { bacteria }^{b}\end{array}$ & $\begin{array}{c}\text { Yersinia } \\
\text { spp. }\end{array}$ & $\begin{array}{c}\text { Pseudomonas } \\
\text { spp. }\end{array}$ & $\begin{array}{c}\text { Alcaligenes } \\
\text { spp. }\end{array}$ & $\begin{array}{l}\text { Micrococcus } \\
\text { spp. }\end{array}$ & $\begin{array}{c}\text { Staphylococcus } \\
\text { spp. }\end{array}$ \\
\hline A & $2.9 \times 10^{5}$ & $1.4 \times 10^{4}$ & $2.0 \times 10^{2}$ & $2.0 \times 10^{2}$ & $5.1 \times 10^{3}$ & $5.0 \times 10^{1}$ & $1.8 \times 10^{5}$ \\
\hline B & $6.7 \times 10^{4}$ & $2.2 \times 10^{4}$ & $N D^{a}$ & ND & $1.9 \times 10^{4}$ & $3.0 \times 10^{2}$ & $2.0 \times 10^{4}$ \\
\hline c & $5.8 \times 10^{2}$ & $1.0 \times 10^{2}$ & ND & ND & ND & ND & $4.0 \times 10^{2}$ \\
\hline D & $4.4 \times 10^{2}$ & ND & ND & ND & ND & ND & $5.0 \times 10^{1}$ \\
\hline$E$ & $1.0 \times 10^{2}$ & ND & ND & ND & ND & ND & ND \\
\hline $\begin{array}{c}\text { Arithmetic } \\
\text { ave. }\end{array}$ & $7.2 \times 10^{4}$ & $1.2 \times 10^{4}$ & $2.0 \times 10^{2}$ & $2.0 \times 10^{2}$ & $1.2 \times 10^{4}$ & $1.8 \times 10^{2}$ & $5.0 \times 10^{4}$ \\
\hline $\begin{array}{c}\text { Geometric } \\
\text { ave. }\end{array}$ & $3.5 \times 10^{3}$ & $3.1 \times 10^{3}$ & $2.0 \times 10^{2}$ & $2.0 \times 10^{2}$ & $9.8 \times 10^{3}$ & $1.2 \times 10^{2}$ & $2.9 \times 10^{3}$ \\
\hline
\end{tabular}

${ }^{a}$ Not detected.

${ }^{\circ}$ Coliform bacteria consisted of Enterobacter spp. and Pantoea spp.

The following were not isolated from the drain ditch of the washing machine by swabbing: Salmonella spp., Shigella spp., Escherichia coli, Serratia spp., Aeromonas spp., Acinetobacter spp., Chryseobacterium spp. and Bacillus spp.

TABLE 7. Conditions related to the washing machine and washing conditions.

\begin{tabular}{ccccccc}
\hline & \multicolumn{3}{c}{ Washing machine } & \multicolumn{3}{c}{ Washing conditions } \\
\cline { 2 - 7 } Sample & $\begin{array}{c}\text { Period of use } \\
\text { (year) }\end{array}$ & Auto or Manual & $\begin{array}{c}\text { Operation time } \\
\text { per day (min) }\end{array}$ & \multicolumn{3}{c}{ Laundering additive $^{a}$} \\
\cline { 3 - 7 } & 4 & Manual & 160 & Detergent & Softener & Bleach \\
B & 5 & Manual & 60 & + & - & + \\
C & 3 & Manual & 30 & + & + & + \\
D & 6 & Auto & 15 & + & + & + \\
E & 7 & Auto & 120 & + & + & + \\
\hline
\end{tabular}

a-, Not performed; + , practiced. 
TABLE 8. Bacteria isolated from bath water that had been left standing for $12 \mathrm{~h}$.

\begin{tabular}{|c|c|c|c|c|c|c|c|c|c|c|}
\hline \multirow[b]{2}{*}{ Sample } & \multirow{2}{*}{$\begin{array}{c}\text { Total } \\
\text { heterotrophic } \\
\text { plate count } \\
(\mathrm{CFU} / \mathrm{ml})\end{array}$} & \multicolumn{9}{|c|}{$\begin{array}{c}\text { Bacterial count } \\
(\mathrm{CFU} / \mathrm{ml})\end{array}$} \\
\hline & & $\begin{array}{l}\text { Coliform } \\
\text { bacteria }^{b}\end{array}$ & $\begin{array}{c}\text { Escheri- } \\
\text { chia } \\
\text { coli }\end{array}$ & $\begin{array}{l}\text { Yersinia } \\
\text { spp. }\end{array}$ & $\begin{array}{c}\text { Pseudo- } \\
\text { monas } \\
\text { spp. }\end{array}$ & $\begin{array}{c}\text { Acineto- } \\
\text { bacter } \\
\text { spp. }\end{array}$ & $\begin{array}{l}\text { Chryseo- } \\
\text { bacterium } \\
\text { spp. }\end{array}$ & $\begin{array}{c}\text { Alcali- } \\
\text { genes } \\
\text { spp. }\end{array}$ & $\begin{array}{l}\text { Staphylo- } \\
\text { coccus } \\
\text { spp. }\end{array}$ & $\begin{array}{l}\text { Bacillus } \\
\text { spp. }\end{array}$ \\
\hline$A$ & $1.4 \times 10^{6}$ & $2.0 \times 10^{2}$ & $\mathrm{ND}^{a}$ & ND & $3.6 \times 10^{3}$ & ND & $5.0 \times 10^{3}$ & $6.4 \times 10^{5}$ & $3.9 \times 10^{2}$ & $8.0 \times 10^{1}$ \\
\hline$B$ & $9.6 \times 10^{6}$ & $9.5 \times 10^{2}$ & $1.1 \times 10^{3}$ & $7.7 \times 10^{2}$ & ND & $4.0 \times 10^{2}$ & $4.8 \times 10^{5}$ & $4.8 \times 10^{5}$ & $1.5 \times 10^{3}$ & $3.0 \times 10^{1}$ \\
\hline C & ND & ND & ND & ND & ND & ND & ND & ND & ND & ND \\
\hline$D$ & $5.0 \times 10^{1}$ & $1.0 \times 10^{0}$ & ND & ND & ND & ND & ND & $1.2 \times 10^{1}$ & $3.1 \times 10^{1}$ & ND \\
\hline$E$ & $2.0 \times 10^{6}$ & $1.5 \times 10^{3}$ & ND & ND & $7.0 \times 10^{1}$ & ND & ND & $1.1 \times 10^{6}$ & $6.4 \times 10^{3}$ & ND \\
\hline $\mathrm{F}$ & $1.2 \times 10^{2}$ & $2.0 \times 10^{0}$ & ND & $1.0 \times 10^{3}$ & ND & ND & ND & $1.0 \times 10^{2}$ & ND & ND \\
\hline $\mathrm{G}$ & $1.4 \times 10^{7}$ & $5.0 \times 10^{2}$ & $5.0 \times 10^{1}$ & ND & $1.5 \times 10^{4}$ & $4.0 \times 10^{3}$ & $3.0 \times 10^{3}$ & $1.2 \times 10^{5}$ & $3.4 \times 10^{4}$ & ND \\
\hline $\mathrm{H}$ & $9.0 \times 10^{5}$ & $1.3 \times 10^{2}$ & ND & ND & ND & $1.0 \times 10^{2}$ & $7.0 \times 10^{3}$ & $2.7 \times 10^{5}$ & $1.2 \times 10^{2}$ & ND \\
\hline I & ND & ND & ND & ND & ND & ND & ND & ND & ND & ND \\
\hline$J$ & $3.8 \times 10^{6}$ & ND & ND & $5.6 \times 10^{5}$ & ND & $3.0 \times 10^{2}$ & $1.0 \times 10^{3}$ & $1.2 \times 10^{5}$ & $1.0 \times 10^{1}$ & ND \\
\hline $\begin{array}{l}\text { Arithmetic } \\
\text { ave. }\end{array}$ & $4.0 \times 10^{6}$ & $4.7 \times 10^{2}$ & $5.8 \times 10^{2}$ & $1.9 \times 10^{5}$ & $6.2 \times 10^{3}$ & $1.2 \times 10^{3}$ & $9.9 \times 10^{4}$ & $3.4 \times 10^{5}$ & $6.1 \times 10^{3}$ & $5.5 \times 10^{1}$ \\
\hline $\begin{array}{c}\text { Geometric } \\
\text { ave. }\end{array}$ & $2.3 \times 10^{5}$ & $8.7 \times 10^{1}$ & $2.3 \times 10^{2}$ & $7.6 \times 10^{3}$ & $1.6 \times 10^{3}$ & $4.7 \times 10^{2}$ & $8.7 \times 10^{3}$ & $3.3 \times 10^{4}$ & $4.7 \times 10^{2}$ & $4.9 \times 10^{1}$ \\
\hline
\end{tabular}

${ }^{a}$ Not detected.

${ }^{b}$ Coliform bacteria consisted of Klebsiella spp., Enterobacter spp. and Pantoea spp.

The following were not isolated from bath water: Salmonella spp., Shigella spp., Serratia spp., Aeromonas spp. and Micrococcus spp.

TABLE 9. Family members and the use of bathing additives.

\begin{tabular}{|c|c|c|c|c|c|c|}
\hline \multirow{3}{*}{ Sample } & \multicolumn{5}{|c|}{ Family member } & \multirow{3}{*}{$\begin{array}{l}\text { Bathing } \\
\text { additive }\end{array}$} \\
\hline & \multicolumn{2}{|c|}{ Male } & \multicolumn{2}{|c|}{ Female } & \multirow{2}{*}{ Total } & \\
\hline & Age & No. & Age & No. & & \\
\hline \multirow[t]{2}{*}{$A$} & 50 's & 1 & 40 's & 1 & 3 & - \\
\hline & & & 20 's & 1 & & \\
\hline \multirow[t]{3}{*}{$\mathrm{B}$} & 40 's & 1 & 40 's & 1 & 4 & + \\
\hline & 20 's & 1 & & & & \\
\hline & 10 's & 1 & & & & \\
\hline \multirow[t]{2}{*}{ C } & 50 's & 1 & 40 's & 1 & 3 & - \\
\hline & 20 's & 1 & & & & \\
\hline \multirow[t]{2}{*}{$D$} & 50 's & 1 & 50 's & 1 & 3 & - \\
\hline & & & 20's & 1 & & \\
\hline \multirow[t]{2}{*}{$E$} & 50 's & 1 & 50 's & 1 & 4 & + \\
\hline & 20 's & 1 & 20's & 1 & & \\
\hline \multirow[t]{2}{*}{$\mathrm{F}$} & 50 's & 1 & 50 's & 1 & 3 & - \\
\hline & & & 20 's & 1 & & \\
\hline \multirow[t]{3}{*}{$G$} & 40 's & 1 & 40 's & 1 & 5 & - \\
\hline & under 10 & 1 & 10 's & 1 & & \\
\hline & & & under 10 & 1 & & \\
\hline \multirow[t]{3}{*}{$\mathrm{H}$} & 50 's & 1 & 70 's & 1 & 4 & - \\
\hline & & & 50 's & 1 & & \\
\hline & & & 20 's & 1 & & \\
\hline \multirow[t]{2}{*}{1} & 30 's & 1 & 20's & 1 & 3 & + \\
\hline & & & under 10 & 1 & & \\
\hline \multirow[t]{3}{*}{ J } & 40 's & 1 & 40's & 1 & 4 & - \\
\hline & 20 's & 1 & & & & \\
\hline & 10 's & 1 & & & & \\
\hline
\end{tabular}

\footnotetext{
${ }^{a}-$, Not added; +, added.
} 
between the composition of family members and the number of isolated bacteria. When the family included a child below the age of 10 , many bacteria were isolated. No correlation was seen between the numbers of isolated bacteria and the addition of bathing additives.

\section{DISCUSSION}

Results from previous studies indicate that laundering operations may be linked to the risk of household microbial transmission (Barrie et al., 1994; Belkin, 2001; Blaser et al., 1984; Buford et al., 1977; Datta and Pridie, 1960; Larson and Duarte, 2001; Oliphant et al., 1949; Sidwell et al., 1971; Standaert et al., 1994; Walter and Schillinger, 1975; Wiksell et al., 1973). The laundering operation in Japan differs from that in the other countries, and the most unique point is the use of the leftover bath water to do the laundry the next day. The occurrence of microbial contamination by following this home laundering practice was investigated.

In the first place, children's underpants were investigated as representative of clothing because we expected the microbial contamination to be remarkable with such clothing. The used bath towel was investigated as a representative of bathroom items which may serve as a bacterial reservoir or disseminator. Furthermore, kitchen rags were investigated as representatives of kitchen items. Many bacteria were isolated from all of these samples (Tables 1-3) and some tendencies were seen in the species of isolated bacteria. Staphylococcus spp. was isolated from all the tested samples, and it was the predominant strain in each sample. Staphylococcus spp. is a representative strain of the microbial flora normally found on human skin, and it is known that some of them might cause microbial infections (Rose and Haas, 1999). Coliform bacteria, Pseudomonas spp., and Bacillus spp. were also isolated from these samples. Contrary to our expectation, very little $E$. coli was isolated from children's underpants (Table 1). We thought that it was because the collected samples were from 4- or 5year-old children, and were not soiled with feces. If the sample had been a baby's underpants or soiled with feces, much more E. coli would have been isolated. Also, a large variation was seen in the number of isolated bacteria from each bath towel (Table 2). Almost the same tendency was seen in a previous report (Scott et al., 1982). The more soiled, damp and smelly the sample was, the more bacteria were isolated (data not shown). Since the soil can serve as a nutrient for bacteria (Terpstra, 1998) and a very humid environment is suitable for bacterial growth, bacterial multiplication would be promoted in samples with such conditions. Therefore, from the viewpoint of home hygiene, much attention is required for the handling of a used bath towel. Many more bacterial species were isolated from kitchen rags ( 8 species) than from the other samples (underpants; 5 species, bath towels; 6 species) (Table 3 ). Similar to the results from bath towels, many bacteria were isolated from the more soiled, damp and smelly samples (data not shown). Generally, the domestic kitchen is one of the places where the risk of bacterial contamination is highest (Scott, 1999; Zhao et al., 1998), and our results support this report. Unlike the results from the United States or European countries, Salmonella spp. was not detected in the kitchen rags. This is thought to reflect the situation that countermeasures against human salmonellosis have been fully realized in Japan. However, other opportunistic pathogens such as Serratia spp. were isolated from the home environment. Moreover, the result that many coliform bacteria were isolated from kitchen rags was also similarly seen in a previous report (Enriquez et al., 1997). Furthermore, an environmental bacterium, Alcaligenes spp., was also isolated from kitchen rags ( 8 of the 10 samples). These results suggest that the crosscontamination from other areas (e.g. a bath or a toilet) to the kitchen utensils might have taken place by a hand contact. For kitchen rags, washing with a detergent is usually performed in many homes (Table 4). But it is obvious that washing with detergents only is inadequate to sterilize kitchen rags. In order to prevent the occurrence of cross-contamination through kitchen rags, the use of boiling water or the addition of bleach is also needed (Scott and Bloomfield, 1990).

We investigated the microbial contamination in the domestic washing machine (Table 5 and 6 ). The contamination of bacteria on the drum and drain ditch was investigated and the occurrence of constant microbial contamination was revealed from our results. Results showed the same tendency found in the results from the public washing machines in the United States (Buford et al., 1977). Many bacteria were isolated from the drain ditch rather than the drum. The reason is thought to be that the drain ditch region is always damp so it is suitable for microbial survival. The kinds of bacteria isolated were generally similar to those isolated from children's underpants, bath towels and kitchen rags. Many Staphylococcus spp. were isolated. This seemed to be natural because Staphylococcus spp. is a representative strain of the normal microbial flora from human skin. Contrary to our expectations, Bacillus spp., which were isolated in great number from children's underpants, were not isolated from the washing machine. When a 
contaminated washing machine is used, not only can perfect laundering hygiene not be attained but also it may cause the cross-contamination from soiled clothes to clean clothes. In the United States or European countries, laundering is often done with a bleach for extra cleaning power (Gibson et al 1999; Racioppi et al., 1994). In Japan, although most of the families used a bleach, many bacteria were isolated (Table 5 and 6 ). This result suggests that the addition of bleach may be effective in cleaning garments but not effective in sanitizing a washing machine.

From bath water, no bacteria were isolated from 2 of the 10 samples contrary to our expectations (Table 8 ). Some of the results showed that many bacteria were isolated though the number of persons using the bath was small. This result indicates the difference in the number of isolated bacteria was due not to the number of persons but the difference in home hygiene. Although many kinds of species were isolated from the other eight samples, they were generally the same types found in the samples from children's underpants, bath towels, kitchen rags and washing machine swabs, except for Alcaligenes spp. which was the predominantly isolated strain. Moreover, the number of isolated bacteria increased in the samples from families in which children of age 10 or younger, or an old person were present (Table 9). Higher levels of hygiene would be needed for these families.

Unlike in many other foreign countries, in Japan it is common for families to use the leftover bath water for the next day's laundry. However, our results revealed that many bacteria existed in the leftover bath water. If this bath water is used to do the laundry, bacterial contamination will occur. The bacteria which were isolated from the bath water were mainly normal environmental microbial flora, so it is thought that they rarely affect our health directly. However, if disease-causing microbes are contained in the used bath water, it is possible that laundering with used bath water may transmit the bacteria to other laundry. Therefore, laundering operations in Japan need to be improved to prevent predictable microbial contamination.

Neither Salmonella spp. nor Shigella spp. was isolated from the investigated samples. This result may reflect that the level of home hygiene in Japan is high. However, it may be unreasonable to describe the level of home hygiene on the basis of our results since samples were collected from only a small part of Japan and only few samples were collected. Moreover, although typical infectious bacteria were not isolated, many bacteria that reveal low levels of hygiene (Bloomfield and Scott, 1997) were isolated. These results suggested the possibility of further microbial contamination and transmission. In order to improve such a situation, we should make efforts to reduce the risk of microbial contamination.

\section{REFERENCES}

Barrie, D., Hoffman, P. N., Wilson, J. A., and Kramer, J. M. (1994) Contamination of hospital linen by Bacillus cereus. Epidemiol. Infect., 113, 297-306.

Belkin, N. L. (2001) Home laundering of soiled surgical scrubs: Surgical site infections and the home environment. Am. J. Infect. Control, 29, 58-64.

Blaser, M. J., Smith, P. F., Cody, H. J., Wang, W. L., and LaForce, F. M. (1984) Killing of fabric-associated bacteria in hospital laundry by low-temperature washing. J. Infect. Dis., 149, 48-57.

Bloomfield, S. F., and Scott, E. (1997) Cross-contamination and infection in the domestic environment and the role of chemical disinfectants. J. Appl. Microbiol., 83, 1-9.

Buford, L. E., Pickett, M. S., and Hartman, P. A. (1977) Sanitation in self-service automatic washers. Appl. Environ. Microbiol., 33, 74-78.

Davis, J. G., Blake, J. R., and Woodall, C. M. (1968) A survey of the hygienic condition of domestic dish-cloths and tea-towels. Medical Officer., 12, 29-32.

Datta, N., and Pridie, R. B. (1960) An outbreak of infection with Salmonella typhimurium in a general hospital. J. Hyg., Camb., 58, 229-240.

Enriquez, C. E., Enriques-Gordillo, R., Kennedy, D. I., and Gerba, C. P. (1997) Bacteriological survey of used cellulose sponges and cotton dishcloths from domestic kitchens. Dairy Food Environ. Sanitat., 17, 20-24.

Finch, J. E., Prince, J., and Hawksworth, M. (1978) A bacteriological survey of the domestic environment. J. Appl. Bacteriol., 45, 357-364.

Gibson, L. L., Rose, J. B., and Haas, C. N. (1999) Use of quantitative microbial risk assessment for evaluation of the benefits of laundry sanitation. Am. J. Infect. Control, 27, S34-S39.

Larson, E. L. (1999) Home hygiene: A reemerging issue for the new millennium. Am. J. Infect. Control, 27, S1-S3.

Larson, E., and Duarte, C. G. (2001). Home hygiene practices and infectious disease symptoms among household members. Public Health Nurs., 18, 116-127.

Meers, P. D., Ayliffe, G. A. J., Emmerson, A. M., Leigh, D. A., Mayon-White, R. T., Mackintosh, C. A., and Stronge, J. L. (1981). Report on the natural survey of infection in hospitals, 1980. J. Hospital Infection, 2 (Suppl.), vii-ix.

Oliphant, J. W., Gordon, D. A., Meis, A., and Parker, R. R. (1949) $Q$ fever in laundry workers, presumably transmitted from contaminated clothing. Am. J. Hyg., 49, 76-82.

Racioppi, F., Daskaleros, P. A., Besbelli, N., Borges, A., Deraemaeker, C., Magalini, S. I., Arrieta, R. M., Pulce, C., Ruggerone, M. L., and Vlachos, P. (1994). Household bleaches based on sodium hypochlorite: Review of acute toxicology and poison control center experience. Food Chem. Toxicol., 32, 845-861.

Rose, J. B., and Haas, C. N. (1999) A risk assessment framework for the evaluation of skin infections and the potential impact of antibacterial soap washing. Am. J. Infect. 
Control, 27, S26-S33.

Sattar, S. A., Tetro, J., and Springthorpe, V. S. (1999) Impact of changing societal trends on the spread of infections in American and Canadian homes. Am. J. Infect. Control, 27, S4-S21.

Scott, E., Bloomfield, S. F., and Barlow, C. G. (1982) An investigation of microbial contamination in the home. J. Hyg. Camb., 89, 279-293.

Scott, E., and Bloomfield, S. F. (1990) Investigations of the effectiveness of detergent washing, drying and chemical disinfection on contamination of cleaning cloths. J. Appl. Bacteriol., 68, 279-283.

Scott, E. (1996) Foodborne disease and other hygiene issues in the home. J. Appl. Bacteriol., 80, 5-9.

Scott, E. (1999) Hygiene issues in the home. Am. J. Infect. Control, 27, S22-S25.

Sidwell, R., Dixon, G., and McNeil, E. (1971) Quantitative studies on fabrics as disseminators of viruses. Part $V$. Effect of laundering on poliovirus-contaminated fabrics. Appl. Microbiol., 21, 227-234.
Standaert, S. M., Hutcheson, R. H., and Schaffner, W. (1994) Nosocomial transmission of Salmonella gastroenteritis to laundry workers in a nursing home. Infect. Control Hosp. Epidemiol., 15, 22-26.

Terpstra, P. M. J. (1998) Domestic and institutional hygiene in relation to sustainability. Historical, social and environmental implications. Int. Biodeterior. Biodegrad., 41, 169175.

Walter, W. G., and Schillinger, J. E. (1975) Bacterial survival in laundered fabrics. Appl. Microbiol., 29, 368-373.

Wiksell, J. C., Pickett, M. S., and Hartman, P. A. (1973) Survival of microorganisms in laundered polyester-cotton sheeting. Appl. Microbiol., 25, 431-435.

World Health Organization. (1998) World Health Report, 1998. World Health Organization Press.

Zhao, P., Zhao, T., Doyle, M. P., Rubino, J. R., and Meng, J. (1998) Development of a model for evaluation of microbial cross-contamination in the kitchen. J. Food Prot., 61, 960-963. 